\title{
Safe discontinuation of nilotinib in a patient with chronic myeloid leukemia: a case report
}

Giovanni Caocci ${ }^{1,2^{*}}$, Marianna Greco ${ }^{1}$ and Giorgio La Nasa ${ }^{1,2}$

\begin{abstract}
Introduction: Although there is a considerable amount of data in the literature on safe discontinuation of first-generation tyrosine kinase inhibitor therapy in patients with chronic myeloid leukemia, little is known about discontinuation of second-generation tyrosine kinase inhibitor therapy. Most previous studies have been focused on dasatinib, and the few cases of nilotinib withdrawal that have been reported had a median follow-up of 12 months. To the best of our knowledge, the present report is the first to describe nilotinib withdrawal with 30 months of follow-up.

Case presentation: We report the case of a 64-year-old Caucasian man diagnosed with chronic-phase chronic myeloid leukemia in April 2005. After 4 years of treatment with imatinib, he became intolerant to the drug and was switched to nilotinib. Two years later, he decided to stop nilotinib. Undetectable molecular response persisted for 30 months after discontinuation of the drug.

Conclusion: Our present case suggests that nilotinib withdrawal is safe for patients with chronic myeloid leukemia who achieve a stable undetectable molecular response. Our patient was homozygous for killer immunoglobulin-like receptor haplotype A, previously reported to be a promising immunogenetic marker for undetectable molecular response. We recommend additional studies to investigate patient immunogenetic profiles and their potential role in complete response to therapy.
\end{abstract}

Keywords: Chronic myeloid leukemia, Imatinib, Killer immunoglobulin-like receptors, Nilotinib, Tyrosine kinase inhibitor discontinuation

\section{Introduction}

One of the most highly debated challenges physicians face when treating patients with chronic myeloid leukemia (CML) is to decide whether tyrosine kinase inhibitor (TKI) therapy can be stopped or must be continued indefinitely. The recent European LeukemiaNet (ELN) guidelines for the management of CML recommend that patients who respond optimally to TKI treatment be continued on it indefinitely at the standard suggested dose [1].

Researchers in several studies have reported safe discontinuation of first-generation TKI imatinib. Overall, these studies show a probability of stable undetectable molecular response (MR) of approximately $40 \%$ at 24 months. Factors associated with sustained response are lower Sokal risk score, duration of imatinib treatment,

\footnotetext{
* Correspondence: giovanni.caocci@unica.it

'Bone Marrow Transplant Center, "R Binaghi" Hospital, Via Is Guadazzonis 3, 09126 Cagliari, Italy

${ }^{2}$ Hematology Unit, Department of Medical Sciences, University of Cagliari, Via Is Guadazzonis 3, 09126 Cagliari, Italy
}

previous interferon therapy, early major MR and duration of undetectable MR [2-4].

However, little is known to date about discontinuation of second-generation TKI therapy. In one study of 34 patients with CML, the investigators reported the feasibility of discontinuing both nilotinib and dasatinib with a median follow-up of 12 months [5]. In a recent preliminary report, researchers described safe withdrawal of dasatinib with a median follow-up of 6 months in 27 patients previously treated with imatinib [6]. Additionally, in three case reports of a total of six patients with median follow-up ranging from 12 to 27 months, the authors showed safe discontinuation in three of the five patients treated with dasatinib and in one patient treated with nilotinib [7-9]. Our report concerns an imatinibintolerant patient who achieved undetectable MR with nilotinib. The patient was in undetectable MR4.5 at 30 months after stopping the drug and remains so at the time of this report. 


\section{Case presentation}

Our patient was a 64-year-old Caucasian man diagnosed with chronic phase CML in April 2005. Peripheral blood tests showed hemoglobin of $13.5 \mathrm{~g} / \mathrm{dl}$, white blood cell count of $17.6 \times 10^{9} / \mathrm{L}$ and platelet count of $402 \times 10^{9} / \mathrm{L}$. No organomegaly was observed. Cytogenetic analysis of the bone marrow aspirate revealed a 46,XY,t(9;22)(q34; q11.2) karyotype in $100 \%$ of metaphases. By qualitative PCR, we detected the sole presence of the b3a2 transcript. According to the patient's Sokal and Hasford scores, he was classified as being in an intermediate risk class. He was started on imatinib $400 \mathrm{mg} /$ day. After 6 months, he had achieved a complete cytogenetic response and MR3 (BCR-ABL1 $\leq 0.01 \%$ ). Four years later, the patient became intolerant to imatinib (grade 3 diarrhea, probably due to the irritant effect of this drug on intestinal mucosa) and was switched to nilotinib. Although the recommended dose for patients resistant or intolerant to imatinib is $800 \mathrm{mg} /$ day, our patient was given $600 \mathrm{mg} /$ day because of residual diarrhea.

He achieved early MR4.5 (BCR-ABL1 $\leq 0.0032 \%$ ) and subsequently undetectable MR4.5, confirmed by nested RT-PCR (kits from Nanogen Advanced Diagnostics, Turin, Italy). The patient decided to stop nilotinib after 24 months of treatment. Undetectable MR4.5 persisted at 30 months after discontinuation of nilotinib and remains so at the time of this report (Figure 1). Investigation of the immune genetic profile of our patient revealed homozygosity for the killer immunoglobulinlike receptor (KIR) haplotype A with the following genes: KIR2DS4, KIR2DL1, KIR2DL3, KIR2DL4, KIR3DL1, KIR3DL2 and KIR3DL3.

\section{Discussion}

Although the ELN 2013 guidelines do not recommend discontinuation of TKI therapy in optimal responders, several attempts have been made to identify patients who can safely stop treatment [1]. The Italian consensus is that TKI discontinuation should be considered only within the context of controlled clinical trials and should be restricted to patients with MR4.5 sustained for at least 2 years. The drug needs to be promptly restarted in patients who lose MR3 [10].

In recent years, more precise definitions of international scale (IS) deep MR have been introduced: MR4 (BCR-ABL1 $\leq 0.01 \%$ ), MR4.5 (BCR-ABL1 $\leq 0.0032 \%$ ) and MR5 (BCR-ABL1 $\leq 0.001 \%)$. An, assay is considered negative when no BCR-ABL copies are detectable using the IS with a sensitivity $\geq 4.5$ logs below the standardized baseline. Indeed, the term complete molecular response is increasingly being replaced with undetectable molecular response [1].

Rea et al., in their study of 34 patients with CML, reported a $58 \%$ probability of maintaining a stable, deep MR at 12 months [5]. Most of their patients had previously been treated with imatinib; patients receiving second-generation TKI treatment because of intolerance to imatinib had a significantly higher probability of achieving stable undetectable MR (67\%) compared to those switched to second-generation TKI because of suboptimal response or resistance (33\%).

Preliminary data on 27 participants enrolled in a larger study of 63 patients with CML who stopped dasatinib after achieving undetectable MR for at least 1 year had a molecular relapse-free survival rate of $44 \%$. Reintroduction of

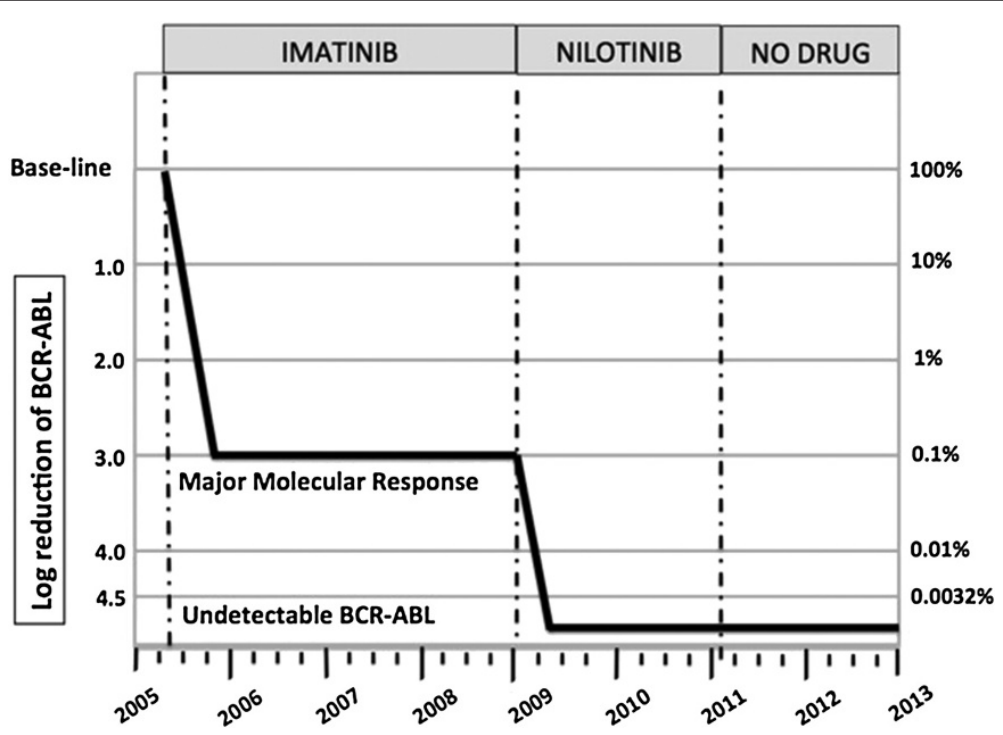

Figure 1 Clinical course of the patient. BCR-ABL levels measured during and after discontinuation of imatinib and nilotinib therapy. 
the drug in relapsed patients rapidly restored deep MR, and none of them progressed to advanced phase CML [6]. However, except for the study by Rea and colleagues [5] and one anecdotal case report [9], both with a median follow-up of only 1 year, there is a paucity of data on withdrawal of nilotinib in patients with CML. Interestingly, our patient was homozygous for KIR haplotype A, previously reported by our research team to be a promising immunogenetic marker for undetectable MR [11]. The authors of another recent report suggest a potential role for natural killer cells in achieving undetectable MR after discontinuation of imatinib [12].

\section{Conclusions}

Our present report, to the best of our knowledge, is the first to describe nilotinib withdrawal with a follow-up of 30 months. Our patient was treated with imatinib for 4 years and achieved MR3 before he was started on replacement therapy with nilotinib because of intolerance. He rapidly achieved undetectable MR and, after 24 months of treatment, decided to stop the drug. He continued to have undetectable MR for 30 months after stopping the drug and remains so at the time of this report. Additional studies are recommended to confirm the feasibility and safety of discontinuing second-generation TKI therapy.

\section{Consent}

Written informed consent was obtained from the patient for publication of this case report and any accompanying images. A copy of the written consent is available for review by the Editor-in-Chief of this journal.

\section{Abbreviations}

BCR-ABL: Breakpoint cluster region-Abelson; CML: Chronic myeloid leukemia; ELN: European LeukemiaNet; IS: International Scale; MR: Molecular response; TKl: Tyrosine kinase inhibitor.

\section{Competing interests}

The authors declare that they have no competing interests.

\section{Authors' contributions}

All authors analyzed and interpreted the patient data and wrote the manuscript. All authors read and approved the final manuscript.

\section{Acknowledgments}

We are grateful to Anna Maria Koopmans (Bone Marrow Transplant Center, R Binaghi Hospital, Cagliari, Italy) for her professional assistance with the writing of the manuscript.

Received: 20 March 2014 Accepted: 21 July 2014

Published: 6 September 2014

\section{References}

1. Baccarani M, Deininger MW, Rosti G, Hochhaus A, Soverini A, Apperley JF, Cervantes F, Clark RE, Cortes JE, Guilhot F, Hjorth-Hansen H, Hughes TP, Kantarjian HM, Kim DW, Larson RA, Lipton JH, Mahon FX, Martinelli G, Mayer J, Müller MC, Niederwieser D, Pane F, Radich JP, Rousselot P, Saglio G, Saußele S, Schiffer C, Silver R, Simonsson B, Steegmann JL, et al: European LeukemiaNet recommendations for the management of chronic myeloid leukemia. Blood 2013, 122:872-884.
2. Mahon FX, Rea D, Guilhot J, Guilhot F, Huguet F, Nicolini F, Legros L, Charbonnier A, Guerci A, Varet B, Etienne G, Reiffers J, Rousselot P, Intergroupe Français des Leucémies Myéloïdes Chroniques: Discontinuation of imatinib in patients with chronic myeloid leukemia who have maintained complete molecular remission for at least 2 years: the prospective, multicentre Stop Imatinib (STIM) trial. Lancet Oncol 2010, 11:1029-1035

3. Ross DM, Branford S, Seymour JF, Schwarer AP, Arthur C, Yeung DT, Dang P, Goyne JM, Slader C, Filshie RJ, Mills AK, Melo JV, White DL, Grigg AP, Hughes TP: Safety and efficacy of imatinib cessation for CML patients with stable undetectable minimal residual disease: results from the TWISTER Study. Blood 2013, 122:515-522.

4. Benjamini $\mathrm{O}$, Kantarjian $\mathrm{H}$, Rios MB, Jabbour E, O'Brien S, Jain $\mathrm{P}$, Cardenas-Turanzas M, Faderl S, Garcia-Manero G, Ravandi F, Borthakur G, Quintas-Cardama A, Cortes J: Patient-driven discontinuation of tyrosine kinase inhibitors: single institution experience. Leuk Lymphoma in press doi:10.3109/10428194.2013.831092.

5. Rea D, Rousselot P, Guilhot F, Tulliez M, Nicolini FE, Guerci-Bresler A, Legros L, Gardembas M, Giraudier S, Guillerm G, Mahon FX: Discontinuation of second generation (2G) tyrosine kinase inhibitors (TKI) in chronic phase (CP)-chronic myeloid leukemia (CML) patients with stable undetectable BCR-ABL transcripts [abstract]. Blood 2012, 120(21 ASH Annual Meeting Abstracts issue):Abstract 916.

6. Tanaka H, Imagawa J, Okada M, Nakamae H, Hino M, Murai K, Ishida Y, Kumagai T, Sato S, Ohashi K, Sakamaki H, Wakita H, Uoshima N, Nakagawa Y, Minami Y, Ogasawara M, Takeoka T, Akasaka H, Morita S, Sakamoto J, Kimura S: Discontinuation of dasatinib in patients with CML who have maintained complete molecular response for at least one year: results from a prospective discontinuation (DADI) trial [abstract]. Am Soc Hematol 2013, 122(21 ASH Annual Meeting Abstracts issue):Abstract 3998.

7. Ross DM, Bartley PA, Goyne J, Morley AA, Seymour JF, Grigg AP: Durable complete molecular remission of chronic myeloid leukemia following dasatinib cessation, despite adverse disease features. Haematologica 2011, 96:1720-1722.

8. Gadó K, Matolcsy A, Csomor J, Kicsi D, Bödör C, Domján G: Long lasting complete molecular remission after suspending dasatinib treatment in chronic myeloid leukemia. Exp Hematol Oncol 2012, 1:17

9. Aoki J, Ohashi K, Kobayashi T, Kakihana K, Hirashima Y, Sakamaki H: Sustained complete molecular response of chronic myeloid leukemia after discontinuation of second-generation tyrosine kinase inhibitors. Leuk Lymphoma 2012, 53:1412-1414.

10. Breccia M, Alimena G, Baccarani M, Bocchia M, Di Raimondo F, Gambacorti Passerini C, Gozzini A, Morra E, Pane F, Pregno P, Rege-Cambrin G, Rosti G, Specchia G, Vigneri P, Saglio G: Current management of CML patients: summary of the Italian Consensus Meeting held in Rome, April 11-12, 2013. Crit Rev Oncol Hematol 2013, 12:17.

11. La Nasa G, Caocci G, Littera R, Atzeni S, Vacca A, Mulas O, Langiu M, Greco M, Orrù S, Orrù N, Floris A, Carcassi C: Homozygosity for killer immunoglobulin-like receptor haplotype A predicts complete molecular response to treatment with tyrosine kinase inhibitors in chronic myeloid leukemia patients. Exp Hematol 2013, 41:424-431.

12. Takahashi N, Kyo T, Maeda Y, Sugihara T, Usuki K, Kawaguchi T, Usui N, Okamoto S, Ohe Y, Ohtake S, Kitamura K, Yamamoto M, Teshima H, Motoji T, Tamaki T, Sawada K, Ohyashiki K: Discontinuation of imatinib in Japanese patients with chronic myeloid leukemia. Haematologica 2012, 97:903-906.

doi:10.1186/1752-1947-8-295

Cite this article as: Caocci et al: Safe discontinuation of nilotinib in a patient with chronic myeloid leukemia: a case report. Journal of Medica Case Reports 2014 8:295. 